\title{
Normal Femorotibial Rotational Alignment and Implications for Total Knee Arthroplasty: an MRI Analysis
}

\author{
Gregory C. Wernecke, MBBS (Hons) • Ian A. Harrris, FRACS (Orth) • Bradley G. Seeto, FRACS (Orth) • \\ Darren B. Chen, FRACS (Orth) • Samuel J. MacDessi, FRACS (Orth)
}

Received: 14 October 2015/Accepted: 11 January 2016/Published online: 22 February 2016

(C) Hospital for Special Surgery 2016

\begin{abstract}
Background: Rotational alignment of prosthetic components in total knee arthroplasty (TKA) is important to successful outcomes. Component malrotation is a known cause of revision and understanding normal rotational alignment may help recreate normal joint kinematics. To date, no large MRI study assessing femorotibial rotational alignment in nonarthritic knees has been undertaken. Questions/ Purposes: Is Insall's tibial axis a reliable rotational landmark against common femoral rotational axes in the nonarthritic patient population? Methods: We reviewed 544 knee MRI scans performed for suspected soft tissue pathology and identified Insall's tibial rotational axis as well as the femoral clinical trans-epicondylar axis (TEAc), femoral surgical trans-epicondylar axis (TEAs), posterior condylar articular axis (PCA), and a modified Eckhoff's cylindrical axis. The perpendiculars of these axes were superimposed on Insall's tibial axis, and the angular differences were measured. Results: Insall's axis was internally rotated to the TEAc by $1.4^{\circ}$, externally rotated to Eckhoff's cylindrical axis by $1.8^{\circ}$, externally rotated to the TEAs by $2.7^{\circ}$, and externally rotated to the PCA by $3.5^{\circ}$. The mean deviation from $0^{\circ}$ (optimal alignment for each femoral axis) was significantly greater for the PCA relative to all other femoral axis. Conclusion: Insall's axis is a reliable landmark for rotational positioning
\end{abstract}

Work performed at Sydney Knee Specialists, Sydney Australia

Level of Evidence: Diagnostic Study Level III

Electronic supplementary material The online version of this article (doi:10.1007/s11420-016-9491-y) contains supplementary material, which is available to authorized users.

G. C. Wernecke, MBBS (Hons) $(\bowtie) \cdot$ I. A. Harris, FRACS (Orth) •

B. G. Seeto, FRACS (Orth) • D. B. Chen, FRACS (Orth) •

S. J. MacDessi, FRACS (Orth)

Sydney Knee Specialists,

8/19 Kensington St,

Kogarah, NSW 2217, Australia

e-mail: dr4734@hotmail.com of the tibial component and may optimize femorotibial kinematics in fixed-bearing TKA.

Keywords knee alignment $\cdot$ rotation $\cdot$ Insall axis · femoro-tibial congruity t tibial component alignment

\section{Introduction}

There are multiple factors that contribute to a successful outcome in total knee arthroplasty (TKA) surgery. The influence of coronal plane positioning on joint survivorship is well documented and has been the major focus of research in attempts to reduce malpositioning and improving outcomes $[1,2,14]$.

More recently, a greater emphasis on rotational positioning of implants in TKA has evolved. Multiple studies have shown that axial malrotation can result in higher revision rates and lower patient-reported outcome scores $[3,7,9]$. Femoral malrotation errors have been documented to result in patellofemoral pain, as well as flexionextension gap imbalance $[9,15,25]$. Less attention has generally been given to axial positioning of the tibial component and its relationship to femoral rotational alignment (femorotibial congruency). However, it is recognized that tibial component internal rotation errors lead to patella maltracking, changes in implant bone coverage and are a cause of revision knee surgery [11, 17, 18, 27]. While exact congruency between femoral and tibial components is not required for a successful outcome, improvements in knee survivorship and increases in patient expectations necessitate a reduction in alignment errors, including axial malrotation. This may be especially true in highly conforming polyethylene implants.

Common femoral surgical landmarks for femoral rotation include the surgical trans-epicondylar axis (TEAs), the clinical trans-epicondylar axis (TEAc), the posterior condylar axis (PCA), and the AP axis (Whiteside's line). More 
Table 1 Cohort characteristics included in study

\begin{tabular}{lll}
\hline Total patient number & Mean age (SD) & Gender distribution (M/F) \\
\hline 544 & $40.4(15.1)$ & $289: 255$ \\
\hline
\end{tabular}

recently, Eckhoff et al. [15] described a femoral flexionextension axis based on cylinders created from posterior femoral condyles. Intraoperatively, tibial component rotation is commonly set according to either tibial anatomic landmarks or articulation with the femoral component. Insall [19] described an anteroposterior (AP) axis from the junction of the medial and middle thirds of the tibial tubercle to the posterior cruciate ligament (PCL). More recently, a computed topography (CT)-based study by Akagi et al. [5] set an AP axis from the medial border of the patella tendon at its tibial insertion citing closer approximation to the femoral surgical TEA. The range of motion (ROM) technique allows the baseplate to rotate on the cut tibial surface and align itself with a trial femoral component. The disadvantage of this technique is that errors in tibial bone coverage may occur due to a lack of set rotational control and tibial tray design $[2,7]$.

These studies of distal femoral and proximal tibial rotational landmarks and subsequent femorotibial alignment have almost exclusively been based on CT imaging and patient cohorts with arthritis and/or deformity [2, 4, $12,13,15,26]$. The operative management of patients with osteoarthritis should be aimed at restoring knee alignment and kinematics to the premorbid state. With this goal in mind, the axial rotational difference between the femur and tibia must be shown in a pathology-free cohort using the articular cartilage surfaces as references. We aim to use MRI to compare Insall's tibial axis to various femoral axes, including a modified cylindrical axis based on the posterior femoral articular cartilage instead of subchondral bone. This will be done to answer the clinical question: Does Insall's AP tibial axis remain a reliable tibial component rotational landmark for femorotibial congruency in the native knee?

\section{Patients and Methods}

This is a retrospective MRI analysis of 544 skeletally mature patients with normal bony anatomy who previously underwent imaging for suspected soft tissue pathology. Patient demographics can be seen in Table 1. Inclusion criteria were patients who underwent investigation for suspected anterior cruciate ligament rupture and/or meniscal pathology. Exclusions were associated fractures, previous open reduction and internal fixation around the knee, multi-ligament knee injuries, and degenerative arthritis (Outerbridge grades 3 and 4). T2-weighted, fat-suppressed MR images (GE Hdx 1.5 T MRI system, General Electric, Waukesha WI) were taken with 3-5-mm thickness with patients in the supine position with the leg in full extension. All measurements were completed using Osirix Dicom Viewer (version 5.5.1) (Osirix Foundation, Geneva).

Insall's tibial axis was recorded as a line from the junction of the medial and middle thirds of the patella ligament to the insertion of the PCL at the level of the tibial resection (Fig. 1). The tibial slice chosen estimated the normal resection level of a TKA as $8 \mathrm{~mm}$ below the lowest point of the lateral tibial plateau. The TEAc was defined as the widest point between the medial and lateral epicondyles of the distal femur. The TEAs was defined as the axis from the medial epicondylar sulcus to the lateral epicondyle. Both the TEAc and TEAs were identified on axial images. The PCA was defined as the most posterior point of the posterior femoral articular cartilage in the same axial image as the TEA. A modification of Eckhoff's cylindrical axis was produced by creating a circle with radius of curvature that matched the articular surface curve of the posterior femoral

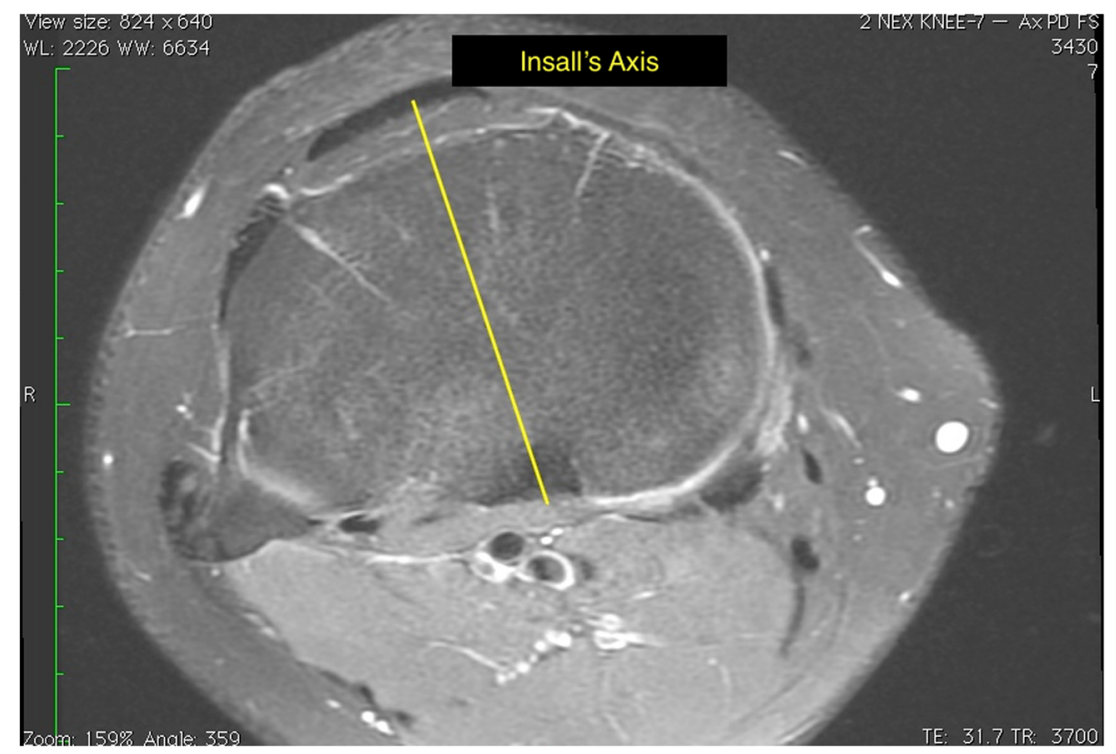

Fig. 1. Axial T2-weighted MRI of the proximal tibia with Insall's axis applied. 


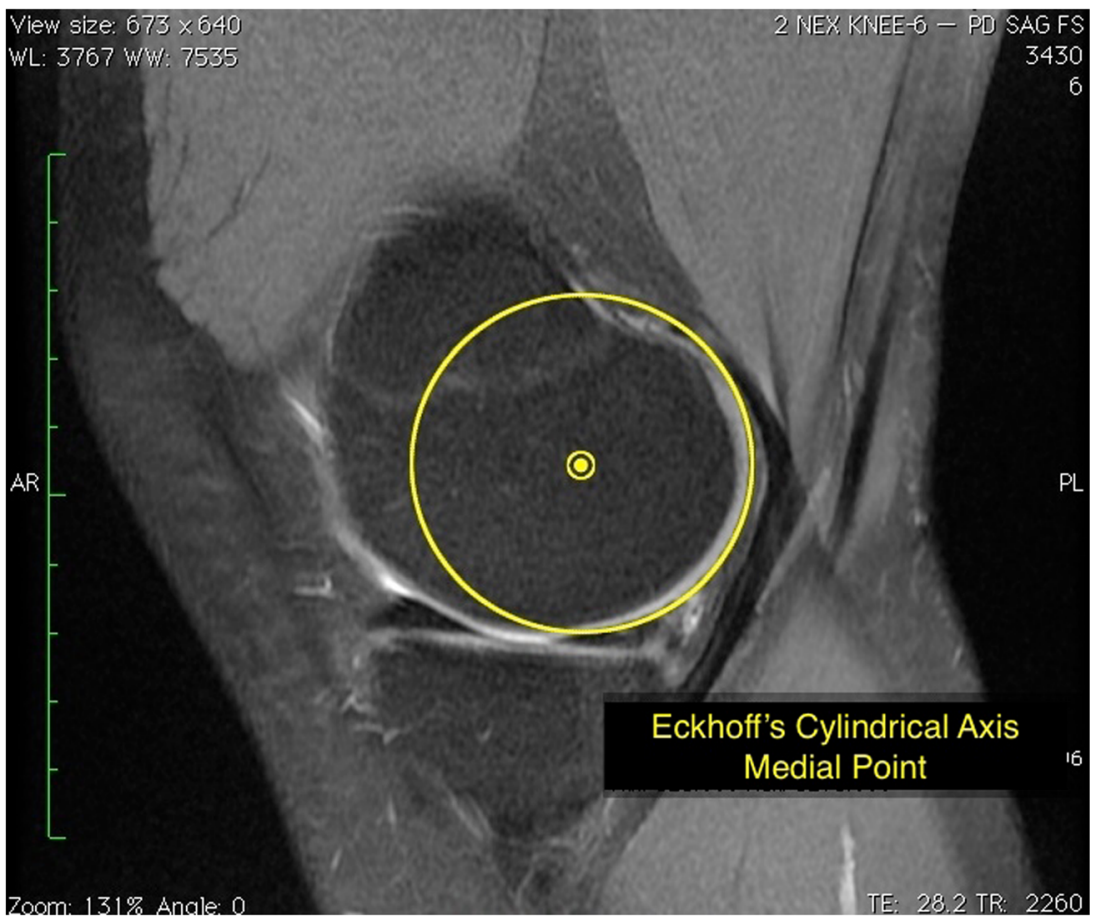

Fig. 2. A sagittal, T2-weighted MRI of the medial compartment of the knee. A circular whose arc of radius matches the articular cartilage arc of the condyle is applied. The center of this circle is the point in which Eckhoff's axis commences. A similar circle is produced for the lateral condyle to allow creation of the flexion/extension axis.

condyle in the sagittal plane on both the medial and lateral sides (Fig. 2). The centers of these circles were transposed to the axial film on both the medial and lateral sides, respectively. A line connecting these points created the rotational axis (Fig. 3).

The four femoral axes were translated distally to the chosen tibial resection slice. The angle between Insall's axis and the perpendicular of each femoral rotational axis was measured (Fig. 4). When Insall's axis was internally rotated to the femoral axis, it was given a positive angular value.
Similarly, external rotation of Insall's axis was given a negative value. Mean angular values and standard deviations (SDs) were recorded. These values landed on both sides of $0^{\circ}$ and so may not give a clear representation of the range of angles. Therefore, the mean difference from $0^{\circ}$ was calculated by using absolute values for each angle, in each group. The mean and SD for these absolute values were then calculated.

Statistical analysis was performed with Microsoft Excel (Microsoft Excel version 14.1; Redmond WA), XLSTAT

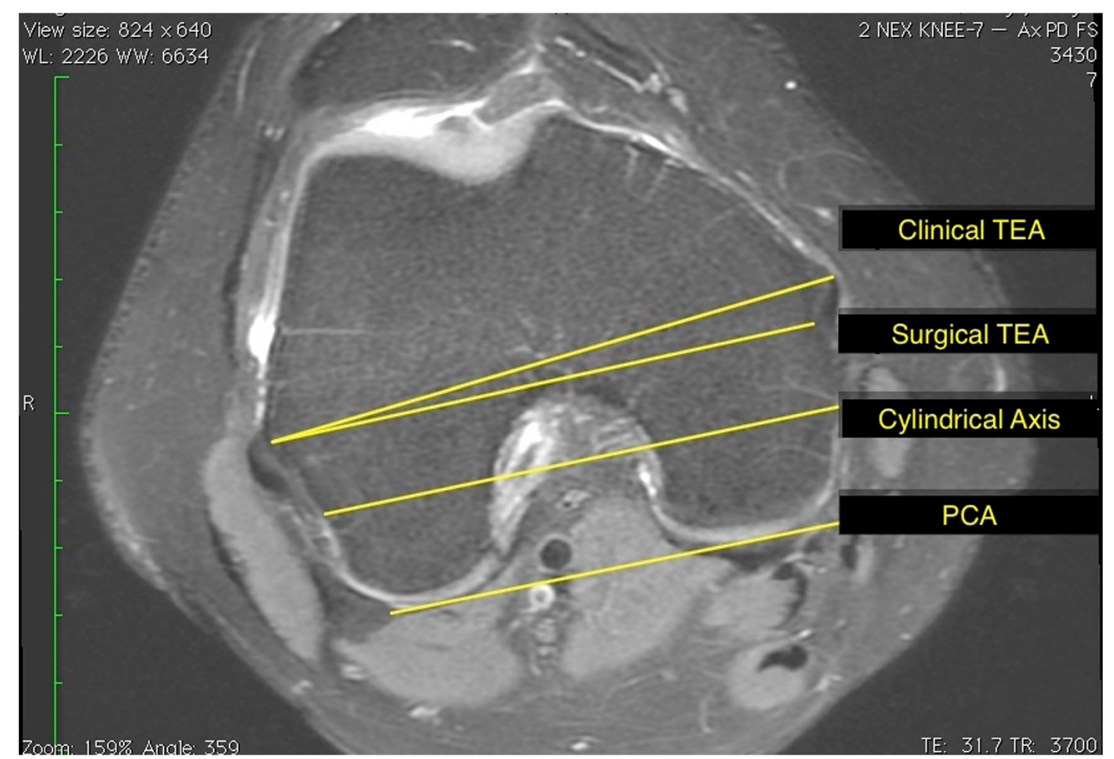

Fig. 3. The distal femoral rotational axes are shown on the axial MRI. 


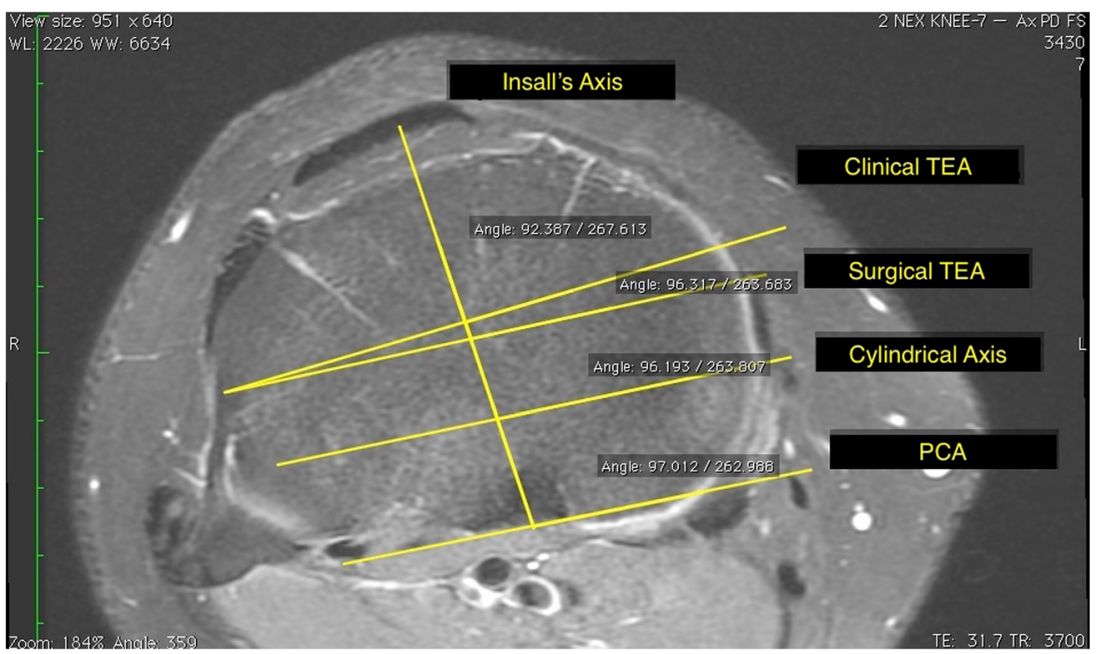

Fig. 4. The femoral rotational axes have been translated distally to the tibial slice where Insall's line has been drawn. The angle between Insall's line and the femoral axes are shown.

(XLSTAT version 2015.3.01, Addinsoft, NY), and STATA 13 (StataCorp, College Station, TX). Shapiro-Wilk testing of data normality failed to show normal distribution. A MannWhitney $U$ test was used to evaluate both the mean angular differences between each Insall-femoral axis angle (e.g., Insall-TEAc vs Insall-TEAs) as well as the mean angular difference from $0^{\circ}$ for the same comparisons. Interobserver reliability of the femoral rotational axis was assessed by randomly choosing 20 patients' scans from our cohort and having three senior authors (GCW, DBC, SJM) independently apply femoral axes. Bland and Altman mean difference and $95 \%$ limits of agreement were performed for each paired comparison.

\section{Results}

Pearson correlation coefficients for interobserver variation showed that all correlations were high, ranging from 0.82 to 0.99 indicating very good to excellent correlations (Table 2).

A medial sulcus was unable to be identified in $5 \%$ of patients $(n=27$, females $=15)$. This left 517 patients to be analyzed in the TEAs group.

Insall's line most closely matched the TEAc and was internally rotated by a mean of $1.4^{\circ}\left(\mathrm{SD} 4.73^{\circ}\right)$. Insall's line was externally rotated to the remaining femoral axes (Table 3). This has been shown graphically in Fig. 5.

Analysis of the mean deviation from $0^{\circ}$ demonstrated that Insall-PCA angle was significantly greater than any other combination (Table 4), and it also had the widest standard deviation.

There was a gender difference such that females had more external rotation of Insall's line relative to men for all femoral axes (Table 5). In women, Insall's axis was still internally rotated to the TEAc.

\section{Discussion}

The goal of this study to was identify the ability Insall's tibial axis to align with common femoral rotational axes in the native knee. We have shown the Insall's axis most closely aligns with the TEAc and least accurately aligns with the PCA.

There are several drawbacks to our study. Firstly, the MRI scans were performed supine and nonweight bearing which does not engage the screw-home mechanism. This may allow for small variations in flexion and rotation of the knee with alterations in femorotibial congruency. Studies using an open MRI scanner and weight-bearing knee joints have clearly shown a change in rotation of the femur on tibia

Table 2 Pearson correlation coefficients of interobserver reliability of axial femoro-tibial angle for three different femoral rotation axes

\begin{tabular}{|c|c|c|c|c|c|c|c|c|c|}
\hline & DR1TEAC & DR2TEAC & DR3TEAC & DR1TEAS & DR2TEAS & DR3TEAS & DR1PCA & DR2PCA & DR3PCA \\
\hline DR1TEAC & 1.0000 & & & & & & & & \\
\hline DR2TEAC & 0.9587 & 1.0000 & & & & & & & \\
\hline DR3TEAC & 0.9587 & 0.9488 & 1.0000 & & & & & & \\
\hline DR1TEAS & 0.9883 & 0.9558 & 0.9532 & 1.0000 & & & & & \\
\hline DR2TEAS & 0.9651 & 0.9718 & 0.9532 & 0.9831 & 1.0000 & & & & \\
\hline DR3TEAS & 0.9367 & 0.9251 & 0.9812 & 0.9538 & 0.9585 & 1.0000 & & & \\
\hline DR1PCA & 0.8692 & 0.8343 & 0.8859 & 0.8500 & 0.8193 & 0.8786 & 1.0000 & & \\
\hline DR2PCA & 0.8806 & 0.8482 & 0.8940 & 0.8699 & 0.8421 & 0.8925 & 0.9942 & 1.0000 & \\
\hline DR3PCA & 0.8952 & 0.8524 & 0.9097 & 0.8663 & 0.8492 & 0.8840 & 0.9531 & 0.9547 & 1.0000 \\
\hline
\end{tabular}

Investigators are identified only by DR1, DR2, and DR3 
Table 3 Comparison of axial femor-tibio angles

\begin{tabular}{lll}
\hline Insall's angular comparison & Mean angle (SD) & $p$ value \\
\hline TEAc : TEAs & $1.4^{\circ}\left(4.7^{\circ}\right):-2.7^{\circ}\left(4.7^{\circ}\right)$ & $<0.0001$ \\
TEAc : Eckhoff & $1.4^{\circ}\left(4.7^{\circ}\right):-1.8^{\circ}\left(4.5^{\circ}\right)$ & $<0.0001$ \\
TEAc : PCA & $1.4^{\circ}\left(4.7^{\circ}\right):-3.5^{\circ}\left(4.8^{\circ}\right)$ & $<0.0001$ \\
TEAs : Eckhoff & $-2.7^{\circ}\left(4.7^{\circ}\right):-1.8^{\circ}\left(4.5^{\circ}\right)$ & $=0.003$ \\
TEAs : PCA & $-2.7^{\circ}\left(4.7^{\circ}\right):-3.5^{\circ}\left(4.8^{\circ}\right)$ & $=0.001$ \\
Eckhoff : PCA & $-1.8^{\circ}\left(4.5^{\circ}\right):-3.5^{\circ}\left(4.8^{\circ}\right)$ & $<0.0001$ \\
\hline
\end{tabular}

with progressive knee flexion $[16,21]$. Secondly, the limb in our study was not held in a particular rotational position for the MRI scan. Uehara et al. [26] used a boot to control rotation in their CT-based study of femorotibial rotation. Using a mix of TEAs and TEAc, they had an average external rotation of $2.6^{\circ}$ to the medial $1 / 3$ of the patella ligament. They also noted an absence of the medial epicondylar sulcus in 29 of 109 patients $(26.6 \%)$. This leads to the third drawback of our study: the variability of the medial epicondylar sulcus. Our intraobserver variability was low for all anatomical sites (and hence axes) with the exception of the medial sulcus. This landmark itself is variable in depth and can be a challenge to accurately palpate intraoperatively. Fourthly, we cannot comment on the coronal alignment of these limbs as they did not have long-leg standing films. Coronal alignment abnormalities such as a valgus knee with a hypoplastic lateral femoral condyle effects measurements using the PCA.

The most reliable method of achieving optimal rotational positioning of prosthetic components is an unresolved issue in total knee arthroplasty. While numerous high-quality studies have been performed in elderly, arthritic cohorts using investigations that lack the ability to truly describe the knee articulation, only a few have been performed using cartilage surfaces as reference points with MRI.

Our literature review only found one paper using MRI to investigate femorotibial rotational alignment in normal

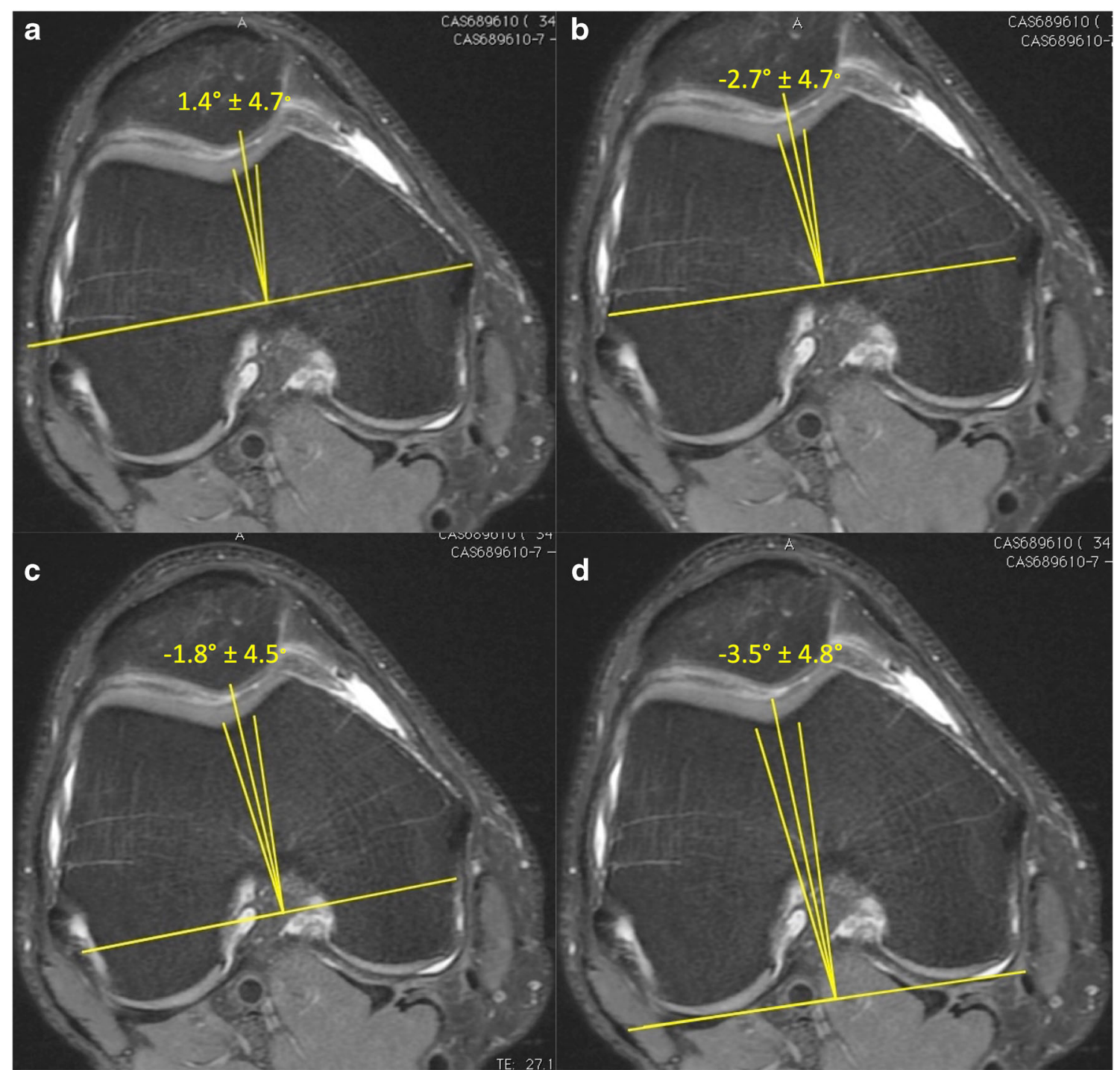

Fig. 5. Mean angular difference $( \pm \mathrm{SD})$ between Insalls tibial axis and each femoral rotational axis (a TEAc, b TEAs, c cylindrical, and d PCA). The longest bisector of each femoral rotational axis is the mean while the shorter lines represent 1 standard deviation in each direction. 
Table 4 In order to better assess the accuracy of the results shown in Table 3, the mean deviation from 0 degrees was calculated by using absolute values of the angles

\begin{tabular}{lll}
\hline Insalls angular comparison & $\begin{array}{l}\text { Absolute angular } \\
\text { departures from } 0^{\circ}\end{array}$ & $p$ value \\
\hline TEAc : TEAs & $3.88^{\circ}: 4.05^{\circ}$ & 0.983 \\
TEAc : Eckhoff & $3.88^{\circ}: 3.68^{\circ}$ & 0.098 \\
TEAc : PCA & $3.88^{\circ}: 4.57^{\circ}$ & $0.006^{\mathrm{a}}$ \\
TEAs : Eckhoff & $4.05^{\circ}: 3.68^{\circ}$ & 0.112 \\
TEAs : PCA & $4.05^{\circ}: 4.57^{\circ}$ & $0.01^{\mathrm{a}}$ \\
Eckhoff : PCA & $3.68^{\circ}: 4.57^{\circ}$ & $<0.0001^{\mathrm{a}}$ \\
\hline
\end{tabular}

This shows that there is no significant difference between TEAc, TEAs, and Eckhoff's axis but that the PCA has significantly more deviation

${ }^{a}$ Statistical significance

knees. Incavo et al. [17] used MRI scans of 30 patients (16 females, mean age 40) to assess the position of a tibial component if it was placed according to the femoral rotational axis and posterior tibial axis. They did not define angular measurements, but described where the short axis of the tibial component would align with the patella ligament. Using the TEAc, $73 \%$ of tibial components pointed within the medial half of the patella ligament. This is similar to our result showing that the clinical epicondylar axis approximates a commonly used tibial rotational axis.

The majority of recent work has come in preoperative cohorts and only studied the femoral rotational landmarks. Patel et al. [23] retrospectively reviewed 557 MRIs performed for preoperative TKA planning (mean age 66.5) to assess femoral rotational axes. They showed that the TEAs was $2.38^{\circ}$ externally rotated to the PCA. In a similar study, Amaranath et al. [6] reviewed 265 MRIbased surgical plans for patient-specific guides and demonstrated a rotational difference of $2.3^{\circ}$ (TEAs externally rotated to PCA). In a smaller preoperative MRI planning study $(n=77)$, Paternostre et al. [24] found the TEAs to be $3.2^{\circ}$ externally rotated to the PCA. Our calculated difference was $0.8^{\circ}$ of external rotation. This may be explained by the presence of arthritic change in these cohorts which may result in asymmetric posterior condylar cartilage loss. Park et al. [22] reviewed 251 preoperative MRIs (mean age 69) using the clinical epicondylar axis and found it to be externally rotated by $6^{\circ}$ to the PCA. These results are larger than our calculated difference of $4.9^{\circ}$. When attempting to restore healthy knee axial alignment, the cartilage appears to play a small but significant role.

We found that females had an Insall's axis that was more externally rotated relative to men for all femoral axes. The gender difference has been shown previously in the earlier anatomical specimen study by Berger et al. [10] who identified a $3.2^{\circ}$ gender difference in PCA versus TEAs.

Insall's tibial axis is a commonly used landmark in total knee arthroplasty. This long-standing rotational axis has served early implants well. As the complexity of prostheses increase (including highly conforming polyethylene, medial pivot and kinematic designs), it is important to reassess the accuracy of both rotational axes and femorotibial congruency. Our study shows that Insall's line remains a reliable landmark that closely approximates the perpendicular of multiple femoral axes. Against all but the TEAc, Insall's line is slightly externally rotated. External rotation of the tibial component improves patellofemoral tracking and avoids the other complications of tibial component internal rotation [11, $17,18,20,27]$.

The complications of component malrotation have helped guide surgeons as to what is clinically acceptable. This is true for both the femoral and tibial components. Berger et al. [9] explored component rotation in patients with patellofemoral pain post-TKA and found that combined internal rotation was more prevalent in this painful group than a matched TKA cohort. Rotational measurements from the TEAs and tibial tubercle showed values ranging from $-1^{\circ}$ to $-17^{\circ}$ of combined internal rotation. It was felt that both components had equal importance in the combined malrotation. Berard et al. [8] focused attention to the tibial component when looking at 50 TKA patients with stiff knees. Their CT analysis showed tibial components were internally rotated by an average of $13.7^{\circ}$ from the center of the tibial tubercle. There is no doubt that TKA is a successful operation, but as the quality of the components continues to increase, it will take long, prospective studies to help identify the subtle aspects of component malrotation to decide what is truly within an acceptable range.

In summary, tibial component rotation is an area of increasing interest. This is the first large study to date to use MRI to analyze Insall's axis in a nonarthritic cohort and compare it to a commonly used tibial anatomical rotational

Table 5 Rotational gender differences of the knee axes

\begin{tabular}{|c|c|c|c|c|}
\hline & $\begin{array}{l}\text { Insall-TEAc angle } \\
\text { Mean (SD) }\end{array}$ & $\begin{array}{l}\text { Insall-TEAs angle } \\
\text { Mean (SD) }\end{array}$ & $\begin{array}{l}\text { Insall-Cylindrical angle } \\
\text { Mean (SD) }\end{array}$ & $\begin{array}{l}\text { Insall-PCA angle } \\
\text { Mean (SD) }\end{array}$ \\
\hline $\begin{array}{l}\text { Male } \\
\text { Female } \\
p \text { value }\end{array}$ & $\begin{array}{l}1.82^{\circ}\left(4.89^{\circ}\right) \\
0.96^{\circ}\left(4.52^{\circ}\right) \\
=0.021\end{array}$ & $\begin{array}{l}-2.33^{\circ}\left(4.39^{\circ}\right) \\
-3.13^{\circ}\left(4.39^{\circ}\right) \\
=0.05\end{array}$ & $\begin{array}{l}-3.13^{\circ}\left(5.0^{\circ}\right) \\
-4.03^{\circ}\left(4.09^{\circ}\right) \\
=0.125\end{array}$ & $\begin{array}{l}-1.49^{\circ}\left(4.56^{\circ}\right) \\
-2.2^{\circ}\left(4.44^{\circ}\right) \\
=0.037\end{array}$ \\
\hline
\end{tabular}

The angle between Insall's line and femoral axes shows that females have greater negative value angles. This could represent external rotation of the tibial tubercle or internal rotation of the distal femoral axes 
axis. We have shown that Insall's tibial axis is a reliable rotational axis with regard to femorotibial congruency when using common femoral rotational axes. Insall's axis is closest to aligning with the TEAc and is externally rotated to all other femoral axes. The posterior condylar axis showed significantly more variation than other femoral axes. Future investigations may compare rotational alignment changes as knee osteoarthritis progresses.

\section{Compliance with Ethical Standards}

Conflict of Interest: Gregory C. Wernecke, MBBS (Hons); Ian A. Harrris, FRACS (Orth); Bradley G. Seeto, FRACS (Orth); Darren B. Chen, FRACS (Orth); and Samuel J. MacDessi, FRACS (Orth) have declared that they have no conflict of interest.

Human/Animal Rights: All procedures followed were in accordance with the ethical standards of the responsible committee on human experimentation (institutional and national) and with the Helsinki Declaration of 1975, as revised in 2008 (5).

Informed Consent: Informed consent was obtained from all patients for being included in the study.

Required Author Forms Disclosure forms provided by the authors are available with the online version of this article.

\section{References}

1. Abdel MP, Oussedik S, Parratte S, Lustig S. Coronal alignment in total knee replacement: historical review, contemporary analysis, and future direction. Bone Joint J. 2014; 96-B(7): 857-862.

2. Aglietti P, Sensi L, Cuomo P, Ciardullo A. Rotational position of femoral and tibial components in TKA using the femoral transepicondylar axis. Clin Orthop Relat Res. 2008; 11: 2751-5.

3. Akagi M, Matsusue Y, Mata T, et al. Effect of rotational alignment on patellar tracking in total knee arthroplasty. Clin Orthop Relat Res. 1999; 366: 155-63.

4. Akagi M, Mori S, Nishimura S, Nishimura A, Asano T, Hamanishi C. Variability of extraarticular tibial rotation references for total knee arthroplasty. Clin Orthop Relat Res. 2005; 436: 172-76.

5. Akagi M, Oh M, Nonaka T, Tsujimoto H, Asano T, Hamanishi C. An anteroposterior axis of the tibia for total knee arthroplasty. Clin Orthop Relat Res. 2004; 420: 213-219.

6. Amaranath JE, Moopanar TR, Sorial RM. Defining distal femoral anatomy for rotational alignment in total knee arthroplasty: a magnetic resonance imaging-based study. ANZ J Surg. 2014. doi:10.1111/ans.12708

7. Barrack RL, Schrader T, Bertot AJ, Wolfe MW, Myers L. Component rotation and anterior knee pain after total knee arthroplasty. Clin Orthop Relat Res. 2001; 392: 46-55.

8. Bedard M, Vince KG, Redfern J, Collen SR. Internal rotation of the tibial component is frequent in stiff total knee arthroplasty. Clin Orthop Rela Res. 2011. Aug;469(8):2346-55
9. Berger RA, Crossett LS, Jacobs JJ, Rubash HE. Malrotation causing patellofemoral complications after total knee arthroplasty. Clin Orthop Rel Res. 1998; 356: 144-53.

10. Berger RA, Rubash HE, Seel MJ, Thompson WH, Crossett LS. Determining the rotational alignment of the femoral component in total knee arthroplasty using the epicondylar axis. Clin Orthop Relat Res. 1993; 286: 40-7.

11. Bindelglass DF. Rotational alignment of the tibial component in total knee arthroplasty. Orthopedics. 2001; 24(11): 1049-1052.

12. Bonnin MP, Saffarini M, Mercier PE, Laurent JR, Carrillon Y. Is the anterior tibial tuberosity a reliable rotational landmark for the tibial component in total knee arthroplasty? J Arthroplasty. 2011 Feb;26)2):260-7

13. Eckhoff DG, Metzger RG, Vandewalle MV. Malrotation associated with implant alignment technique in total knee arthroplasty. Clin Orthop Relat Res. 1995; 321: 28-31.

14. Fang DM, Ritter MA, Davis KE. Coronal alignment in total knee arthroplasty: just how important is it? J Arthroplasty. 2009; 24(6 Suppl): 39-34.

15. Hancock CW, Winston MJ, Bach JM, Davidson BS, Eckhoff DG. Cylindrical axis, not epicondyles, approximates perpendicular to knee axes. Clin Orthop Relat Res. 2013; 471(7): 2278-83.

16. Hill PF, Vedi V, Williams A, Iwaki H, Pinskerova V, Freeman MAR. Tibiofemoral movement 2: the loaded and unloaded living knee studied by MRI. J Bone Joint Surg (Br). 2000; 82-B: 11961198.

17. Incavo SJ, Coughlin KM, Pappas C, Beynnon BD. Anatomic rotational relationships of the proximal tibia, distal femur, and patella: implications for rotational alignment in total knee arthroplasty. J Arthroplasty. 2003; 18(5): 643-8.

18. Incavo SJ, Wild JJ, Coughlin KM, Beynnon BD. Early revision for component malrotation in total knee arthroplasty. Clin Orthop Relat Res. 2007. May;458:131-6

19. Insall JN. Surgical technique and instrumentation in TKA. In: INsall JN, ed. Surgery of the Knee. New York NY: Churchill Livingstone: 1993;739-893

20. KimYH PJW, Kim JS, Park SD. The relationship between the survival of total knee arthroplasty and postoperative coronal, sagittal and rotational alignment of knee prosthesis. Int Orthop. 2014; 38(2): 379-85.

21. Nakagawa S, Kadoya Y, Todo S, et al. Tibiofemoral movement 3: full flexion in the living knee studied by MRI. J Bone Joint Surg (Br). 2000; 82-B: 1199-1200.

22. Park IS, Ong A, Nam CH, et al. Transepicondylr axes for femoral component rotation might produce flexion asymmetry during total knee arthroplasty in knees with proximal tibia vara. Knee. 2014; 21(2): 369-73.

23. Patel AR et al. Femoral component rotation in total knee arthroplaty: an MRI-based evaluation of our options. $J$ Athroplasty. 2014. doi:10.1016/j.arth.2014.02.033.

24. Paternostre F, Schwab PE, Thienpont E. The combined Whiteside's and posterior condylar line as a reliable reference to describe axial distal femoral anantomy in patient-specific instrument planning. Knee Surg Sports Traumatol Arthrosc. 2014. doi:10.1007/s00167-014-2836-5.

25. Romero J, Stahelin T, Binkert C, Pfirrmann C, Hodler J, Kessler $\mathrm{O}$. The clinical consequences of flexion gap asymmetry in total knee arthroplasty. J Arthroplasty. 2007; 22(2): 235-40.

26. Uehara K, Kadoya Y, Kobyashi A, Ohashi H, Yamona Y. Bone anatomy and rotational alignment in total knee arthroplasty. Clin Orthop Relat Res. 2002; 402: 196-201.

27. Wernecke GC, Harris IA, Houang M, Seeto BG, Chen DB, MacDessi SJ. Comparison of tibial bone coverage of 6 knee prostheses: a magnetic resonance imaging study with controlled rotation. J Orthop Surg. 2012; 20(2): 143-7. 